Kozyra Magdalena, Zimnicki Patryk, Kaczerska Justyna, Śmiech Natalia, Nowińska Martyna, Milanowska Joanna. The effect of vitamin D on mental health - literature analysis. Journal of Education, Health and Sport. 2020;10(8):398-407. eISSN 2391-8306. DOI http://dx.doi.org/10.12775/JEHS.2020.10.08.048

https://apcz.umk.pl/czasopisma/index.php/JEHS/article/view/JEHS.2020.10.08.048

https://zenodo.org/record/4007105

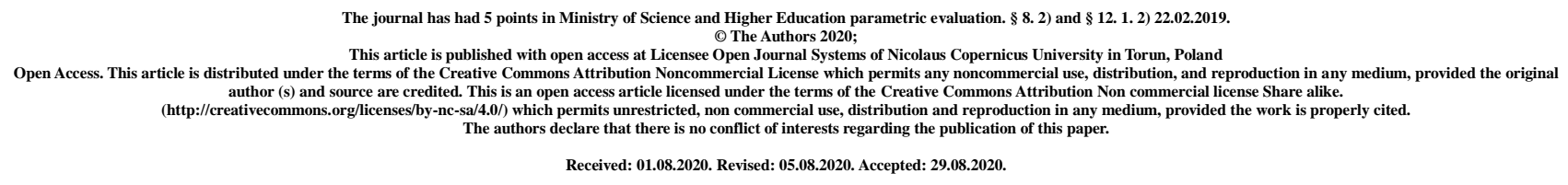

\title{
The effect of vitamin D on mental health - literature analysis
}

\author{
Magdalena Kozyra ${ }^{1}$, Patryk Zimnicki ${ }^{1}$, Justyna Kaczerska $^{1}{ }^{1}$ Natalia Śmiech $^{1}$, \\ Martyna Nowińska ${ }^{1}$, Joanna Milanowska ${ }^{2}$
}

(1) Student Research Group of Applied Psychology, Medical University of Lublin

(2) Department of Applied Psychology, Medical University of Lublin

*Address for correspodence: Staffa 24, 20-454 Lublin, telephone number: 695562174, e-mail address: madzia.kozyra.1221@ poczta.onet.pl

\section{ORCID ID and E-mail}

Magdalena Kozyra https://orcid.org/0000-0002-0260-4102 madzia.kozyra.1221@ poczta.onet.pl

Patryk Zimnicki https://orcid.org/0000-0002-5808-8661 patryk.zimnicki.97@o2.pl

Justyna Kaczerska https://orcid.org/0000-0003-2642-9377 justynakaczerska@ gmail.com

Natalia Śmiech https://orcid.org/0000-0002-8920-7969 natalia.smiech@gmail.com

Martyna Nowińska https://orcid.org/0000-0002-8167-698X m.nowinska98@gmail.com

Joanna Milanowska https://orcid.org/0000-0001-9741-1583 joannamilanowska@umlub.pl 


\section{ABSTRACT}

Introduction: Many sources list a number of properties of vitamin D on human health. It seems that the benefits of using this vitamin include not only the regulation of the calcium-phosphate metabolism, but also a number of other applications, also in human mental health.

The aim of the study: The aim of the study was to research about the influence of vitamin D on the mental health. The research was done on the problems such as depression, anxiety, psychoses, stress, mood and emotions.

Material and method: The literature was found using websites such as PubMed or Google Scholar was used to develop this work.

Description of the state of knowledge: A number of studies have confirmed that vitamin D can have a significant impact on reducing the level of depression. One example of a study on this topic is that high vitamin D supplementation in women in early pregnancy can protect them from late depression during the last weeks of pregnancy. Likewise, adequate vitamin D supplementation in older people can improve mental health and reduce the likelihood of depression and Seasonal Mood Disorder (SAD). In addition, vitamin D can reduce the probability of psychosis or anxiety disorders.

Summary: Many sources report a positive correlation between vitamin D supply and mental health. Often the differences are also noticeable, but not statistically significant. This work sometimes shows divergent views of scientists, which is important to continue research into the effects of vitamin D on mental health in humans.

Keywords: vitamin D; anxiety; depression; psychoses; stress; mood

\section{Introduction}

Vitamin D has always been considered as a factor responsible for maintaining the calciumphosphate balance in the body and the structure of the skeleton. It has been known for about 20 years that this vitamin also plays other functions in the body.

The basic action of vitamin $\mathrm{D}$ is participation in the processes of development and growth and maintaining the proper structure of the skeleton by regulating the calcium metabolism in the body. It happens as a result of the increased absorption of calcium ions in the intestines and their reabsorption in the kidneys [1].

In recent years, there has been an increased interest in the so-called non-classical action of vitamin $\mathrm{D}$ on the human body. Vitamin $\mathrm{D}$ has been found to be involved in other biochemical mechanisms in the body. Lowering its concentration may increase the risk of developing many diseases. Numerous studies suggest a relationship between vitamin D deficiency and the occurrence of diseases such as cancer (colorectal cancer, breast cancer), hypertension, diabetes, metabolic syndrome, multiple sclerosis [2]. Vitamin D works through its VDR receptors, which are located in many organs: bones, skeletal muscles, cells of the immune system, and the brain.

Vitamin D is a group of steroids, of which the most important for humans are vitamin D2 (ergocalciferol) and D3 (cholecalciferol), which differ in the structure of the side chain.

It is synthesized mainly in the skin, from which $80 \%$ of this compound in the body comes from. Many external factors and general health affect the production of cholecalciferol. 7-dehydrocholesterol (provitamin D3) contained in keratinocytes is transformed into cholecalciferol, i.e. vitamin D3. 
This product becomes biologically active only after two hydroxylations. The first of them occurs in the liver, and the reaction catalyst is vitamin D 25-hydroxylase. The end product of the reaction is 25-hydroxyvitamin D3-25 (OH) D3-calcidiol. It is released into the bloodstream and transported in a complex with DBP (vitamin D-binding protein). The transformation of calcidiol into the most biologically active form - calcitriol [1,25 (OH) 2D3] takes place in the renal tubular cells. The reaction is catalyzed by the mitochondrial 25-hydroxyvitamin D 1-hydroxylase [3].

Researchers do not agree on the correct concentration of vitamin. D in blood. Some believe that the optimal concentration of calcitriol is the one that has an appropriate effect on calcium resorption in the intestines and its increase does not intensify this phenomenon $(32 \mathrm{ng} / \mathrm{ml}$ ), others that it is the maximum concentration resulting from skin photosynthesis $(60 \mathrm{ng} / \mathrm{ml})$. According to the latest research, it is assumed that the most advantageous is to maintain the vitamin D concentration in the range of 30$80 \mathrm{ng} / \mathrm{ml}$. The values of $20-30 \mathrm{ng} / \mathrm{ml}$ indicate hypovitaminosis, $10-20 \mathrm{ng} / \mathrm{ml}$ - deficiency, and the concentration below $10 \mathrm{ng} / \mathrm{ml}$ - clearly deficient $[4,5]$.

Vitamin D crosses the blood-brain barrier, but is also synthesized in the brain. Calcitriol, influencing the expression of numerous genes, regulates the synthesis of their products, such as: neurotropins, growth factors, calcium-binding proteins, oxytocins and enzymes - acetylcholinesterase responsible for the synthesis of acetylcholine and gammaglutamyltranspeptidase [6]. Modern research suggests that vitamin D metabolites may influence the behavior of cognitive functions through their specific neuroprotective effects. People with lowered vitamin D levels achieve worse results in tests examining cognitive functions [7]. There is also immunohistochemical, biochemical, and molecular evidence that vitamin D contributes to brain development and function. The authors of many studies report the deterioration of the brain's work with improper supply of micronutrients [8-11], other researchers indicate the improvement of cognitive functions in people whose vitamin and micronutrient concentration were normal [12-14].

\section{Materials and methods}

The data for the article was found using the PubMed and Google Scholar websites. They have been thematically selected for work. Articles were searched using key words such as: vitamin D; depression; anxiety; psychosis; bipolar disease; stress; mood; emotions.

\section{Depression}

Taking into account the influence of vitamin D on mental health, it is impossible not to mention a few of the most important diseases that are impossible not to meet in your life (not only in the professional aspect, but also in private life). One of these diseases is depression.

According to WHO, the effects of depression are felt by about 300 million people worldwide [15]. The pathophysiological causes of depression are disorders related to neurotransmitters such as deficiencies in norepinephrine, serotonin, dopamine, etc. [16]. In addition, there are many theories focusing on psychoanalysis and the impact of, for example, behavioral models or cognitive models on depression [17]. A number of studies have also looked at the effects of vitamin D on depression and depressive symptoms.

It turns out that not all research results unanimously indicate the effect of vitamin $\mathrm{D}$ on depression. One review study looked at thirty studies. 
Mostly it has been confirmed that low blood levels of vitamin D may contribute to late-life depression [18]. In pregnant women, the level of vitamin D and its effect on depression are also proven. It has been confirmed that low vitamin D levels at the beginning of pregnancy (12-20 weeks of gestation) may predispose patients to depression in the final weeks of pregnancy (34-46 weeks of gestation). However, it was not investigated whether early vitamin D supplementation would have an impact on the prevention of this depression [19]. If you look at people classified as having major depressive disorder (MDD) and their vitamin D levels, it turns out that the higher their Hamilton Rating Scale for Depression (HAMD) score, the lower their vitamin D blood levels. detected. Moreover, a negative correlation was observed in relation to total intracranial volume (TIV). TIV itself showed a positive correlation with vitamin D levels in patients with MDD [20].

Since it is known that lowered vitamin D levels are common in elderly people with depressive symptoms, attempts have been made to determine whether vitamin D supplementation can reduce the symptoms of Seasonal Mood Disorder (SAD). The results of the research are ambiguous, as it turns out that supplementation only in some patients has a positive effect on the mental state. Perhaps the improvement of the mental state depends on the administered dose of vitamin D.

After all, there are studies that disprove other studies and state that overall vitamin D supplementation does not relieve symptoms of depression. However, there are discrepancies in the effect of depression depending on the severity of clinical symptoms of depression. In severely symptomatic patients, a slight statistically significant effect was observed, and in asymptomatic patients, a very small and nonsignificant effect of vitamin D supplementation on depression [21] was observed.

\section{Psychoses}

Psychoses themselves are rare among humans and it is reported that only about $10 \%$ of the population has experienced at least one episode of psychosis [22]. Schizophrenia is caused not only by genetics, but also by education, or even lifestyle - a bad diet may contribute to the risk of schizophrenia [23]. It is also worth remembering that psychoses are identified not only with schizophrenia, but also with bipolar disorder, in which mania phases interrupted by phases of psychoses can be distinguished. Single cases of psychosis alone can occur independently of these two diseases and may never even be diagnosed by a psychiatrist.

And just like a good diet, such an abundant supply of vitamin D can lower the likelihood of psychotic-like symptoms in women, as a study of over 33,000 Swedish women has confirmed. And although this study was distinguished not only by the supply of vitamin D, but also the amount of fish meals and PUFA consumed per week. All these issues are inextricably linked with the supply of vitamin D. For example, a diet rich in fish (eating them 3-4 times per week) significantly reduced the risk of psychosis. A similarly high supply of omega-3 and omega-6 PUFAs in the diet compared to a diet with a reduced fatty acid content also lowered the risk of psychosis [24]. Another female study that demonstrated the effects of vitamin D on psychotic experiences (PEs) was one conducted in over 34,000 middle-aged women who tested association between the sun exposure and PEs. As mentioned at the beginning, sun exposure increases the production of vitamin D by UVB radiation, and thus increases the level of this vitamin. Women who tan quite often had a lower score on community assessment of psychic experiences (CAPEs). 
Similarly, in women with a negligible history of sunbathing, the CAPEs results were significantly higher than in women sunbathing in their youth (10-39 years) [25]. It is worth noting that the level of vitamin $\mathrm{D}$ and episodes of psychosis does not only concern women, but the general public, which is confirmed by numerous studies.

\section{Anxiety}

Research shows that many factors can influence the occurrence of anxiety. The literature mentions the effect of diet on anxiety. With obesity, anxiety levels may increase, but the use of metformin, which lowers body weight, and act on AMPK pathway has been shown to have fewer symptoms of anxiety in rats [26]. An unhealthy diet, rich in fat, lard, or sucrose also showed a positive correlation with the level of anxiety. Among the four diets studied by Salehi-Abargouei A. et al. the first containing a large amount of amino acids, cobalamin, zinc, phosphorus, saturated fatty acids, cholesterol and pantothenic acid, turned out to be the best in reducing anxiety [27]. Scientists have also studied the effect of vitamin D levels on this mental disorder.

In children with chromosome 22q11.2 deletion syndrome (22q11.2DS), anxiety and atypical behavior were observed in relation to their healthy peers. A one-tailed test was used which showed that those of the children who had lower vitamin D levels had higher levels of anxiety. However, the level of vitamin D was not associated with atypical behavior [28]. Another study on anxiety and vitamin D was conducted in children and adolescents undergoing hemodialysis. The same correlation was also found in them, which shows that low vitamin D levels may favor the appearance of anxiety disorders in this group of patients [29].

Depression and anxiety were also studied in pregnant women using Depression, Anxiety, and Stress Scales (DASS-21) and Patient Health Questionnaire Depression Module (PHQ-9) compared to this level of vitamin D. It turned out that in those women who had the lowest vitamin D levels, had 1.11 higher PHQ-9 scores than those with the highest vitamin D levels. However, this result did not turn out to be statistically significant [30].

In another retrospective study, the studied Koreans were divided into three groups depending on the concentration of vitamin D in the body. Adequate vitamin D levels were found above $\geq 20 \mathrm{ng} / \mathrm{mL}$, inadequate between 10-19.99 $\mathrm{ng} / \mathrm{mL}$, and vitamin D deficiency below <10 ng / mL. A correlation was found between the occurrence of anxiety states in the respondents depending on the level of vitamin D. The higher its level, the lower the probability of anxiety states [31].

\section{Stress}

As another determinant of mental health, stress has also been the subject of much research. Its relationship to the amount of vitamin $\mathrm{D}$ in the blood was also checked. One of the larger randomized clinical trials has investigated many relationships between vitamin D and, for example, depression and anxiety. It also assessed the intensity of stress in the respondents' lives. However, this was not related to any degree with vitamin D levels [32].

One study focused on low vitamin D levels as a predisposing factor to myocardial ischemia. They checked whether low levels could induce Mental stress-induced (MSIMI) or physical stressinduced (PSIMI) myocardial ischemia. The study confirmed that vitamin D deficiency can cause MSIMI but has no effect on PSIMI [33]. 


\section{Mood and emotional state}

Mood also contributes to your the mental health. The hallmark of good mental health is a positive mood and possibly a rarely depressed, negative mood.

An older study was the one in which they wanted to see if the seasonal depressed mood resulting from the winter period could be mitigated with vitamin D supplementation. Although the experience was short, as the subjects received a dose of vitamin D for five days, the researchers found a positive correlation between vitamin D intake and a positive effect on the mood of the respondents [34].

In one more recent double-blind study, 152 women were examined to find out about the number of links between vitamin $\mathrm{D}$ and mental health. No correlation was found between vitamin D supplementation (in the amount of 50,000 IU every month for six months) and more days with a good mood or fewer days with a worse mood, compared to the control group [35]. In 2017, Penckofer et al. published their study in which it is interesting that the better effect of vitamin D supplementation on mood is observed when the respondents do not take mood-modulating drugs (antidepressants or anxiolytics) [36].

There were also fewer emotional problems in the group of children and adolescents with higher vitamin D. Interestingly, fewer problems with peer relationships were also observed in this group [37]. All this also proves the positive influence of this vitamin on mental health. However, Dean et al. Came to conflicting conclusions, and proved that vitamin D supplementation did not affect cognitive nor emotional functioning in adult humans [38]. The question is whether the age or other factor of the respondents may be of importance in these two contradictory studies?

\section{Conclusion}

Vitamin D is gaining popularity all the time. Not only among people who often and willingly use it, but also among scientists who pay close attention to its properties not only on the body's calcium-phosphate balance, but also on its effects in psychiatric diseases.

A number of studies talk about the effects of vitamin D in depression, anxiety, mood, and more.

The most comprehensive literature is that covering knowledge and research on the effects of vitamin $\mathrm{D}$ on depression. It is said that maintaining an adequate level of vitamin D may prevent latelife depression in humans [18], and good supplementation in the first weeks of pregnancy may have a preventive effect on the occurrence of postpartum depression [19].

In an experiment conducted by English researchers, during which 800 IU of vitamin D was administered to women over 70 years of age, no improvement in mood was noted in the study group [39]. Another study concerned SAD and D hypovitaminosis. American scientists examined 15 people diagnosed with SAD. One group received vitamin D in a dose of 100,000 IU, and the other group received phototherapy (wavelength 280-320 nm). Mood was assessed with the Hamilton Depression Scale at the beginning and after one month of treatment. There was an improvement in the presence and severity of depressive symptoms in all patients receiving vitamin $\mathrm{D}$, while no significant improvement was noted after the use of phototherapy [40]. There is no doubt, however, that vitamin D has a beneficial effect on our body. By taking care of its proper level in the body, we reduce the risk of osteoporosis, take care of the bones and proper calcium management. 
Although there is no consensus among researchers about the influence of vitamin D on mental health, there are some, that prove its positive impact in this aspect, which is another argument encouraging its proper supply of this substance.

When it comes to the effect of vitamin D on anxiety, here we have a lot of confirmations in the studies, although not all of them are statistically significant, as in the case of women in whom the level of anxiety at the lowest values of vitamin D was higher than in those with the highest levels. Unfortunately, although the difference was noticed, it was too small to conclude a statistically significant correlation [30]. Much less research has investigated the relationship between vitamin D levels/supply and stress. The ones found did not notice any correlation. On the other hand, when we take into account myocardial ischemia and the cause of its formation (the study differentiated between MSIMI and PSIMI), it turns out that vitamin D levels have an influence on MSIMI and none with PSIMI, which may prove the relationship between vitamin D and stress as a cause. to the formation of myocardial ischemia [33].

Also, the above studies cited in this paper show that vitamin D may have an influence on some mental states in humans. Relationships were also often observed, although too small to be statistically significant. Since vitamin D has a significant impact on other aspects of human health in addition to mental health aspects, it is worth encouraging patients to be adequately supplemented. It is also worth focusing on educating patients about the appropriate therapeutic dose. The upper limit of the daily intake of vitamin D is about 2,000 IU/day [41]. However, there are patients who reach for dietary supplements with doses of up to 50,000IU/day due to the inappropriate influence of third parties. Such high doses are associated with a rapid increase in vitamin D levels, even up to $150 \mathrm{ng} / \mathrm{mL}$, which is considered as a toxic dose. Rare but serious complications of vitamin D overdose and intoxicity are primarily hypercalcemia and hypercalciuria [42], which may further lead to: muscle weakness, renal dysfunction, calcium deposits in soft tissues, arrhythmias or calcification of the vessels and heart valves, leading to subsequent dysfunctions of the cardiovascular system [43].

Therefore, it is always worth remembering about the good sides of using vitamin D preparations, and also to avoid side effects resulting from an overdose of this vitamin. However, overdosing is not possible with judicious use of the recommended doses.

\section{References}

[1] Holick M.F.: Vitamin D and bone health. J. Nutr. 1996; 126 (supl.): 1159S-1164S

[2] Woźniacka A., Bogaczewicz J., Sysa-Jędrzejowska A.: Drugie oblicze słońca. Prawdziwy „D”ylemat. Część 1. Przegl. Dermatol. 2008; 95: 467-474

[3] Haddad J.G., Matsuoka L.Y., Hollis B.W. i wsp.: Human plasma transport of vitamin D after its endogenous synthesis. J. Clin. Invest. 1993; 91: 2552-2555

[4] Segaert S., Bouillon R.: Vitamin D and regulation of gene expression. Curr. Opin. Clin. Nutr. Metab. Care 1998; 1: 347-354.

[5] NAS/DRI: Dietary Reference Intakes for Calcium, Phosphorus, Magnesium, Vitamin D, and Fluoride. National Academy Press, Washington, D.C. 1997

[6] Romer T.E.: Endokrynologia kliniczna dla ginekologa, internisty i pediatry. Springer PWN, Warszawa 1998. 
[7] Przybelski R.J., Binkley N.C.: Is vitamin D important for preserving cognition? A positive correlation of serum 25-hydroxyvitamin D concentration with cognitive function. Arch. Biochem. Biophys. 2007; 460: 202-205.

[8] Bryan J., Osendarp S., Hughes D. i wsp.: Nutrients for cognitive development in school-aged children. Nutr. Rev. 2004; 62: 295-306.

[9] McCann J.C., Ames B.N.: Is docosahexaenoic acid, an n-3 long-chain polyunsaturated fatty acid, required for development of normal brain function? An overview of evidence from cognitive and behavioral tests in humans and animals. Am. J. Clin. Nutr. 2005; 82: 281-295.

[10] McCann J.C., Hudes M., Ames B.N.: An overview of evidence for a causal relationship between dietary availability of choline during development and cognitive function in offspring. Neurosci. Biobehav. Rev. 2006; 30: 696-712.

[11] McCann J.C., Ames B.N.: An overview of evidence for a causal relation between iron deficiency during development and deficits in cognitive or behavioral function. Am. J. Clin. Nutr. 2007; 85: 931945.

[12] Siega-Riz A.M., Popkin B.M.: Dietary trends among low socioeconomic status women of childbearing age in the United States from 1977 to 1996: a comparison among ethnic groups. J. Am. Med. Womens Assoc. 2001; 56: 44-48, 72.

[13] Lee J.S., Frongillo E.A. Jr: Nutritional and health consequences are associated with food insecurity among U.S. elderly persons. J. Nutr. 2001; 131: 1503-1509.

[14] Dixon L.B., Winkleby M.A., Radimer K.L.: Dietary intakes and serum nutrients differ between adults from food-insufficient and food-sufficient families: Third National Healthand Nutrition Examination

[15] World Health Organization (WHO) (2017). Depression. Fact sheet. Available online at: http://www.who.int/mediacentre/factsheets/fs369/en/

[16] Narbona J. Fenomenología depresiva al inicio de enfermedades neuropediátricas [Depressive phenomenology at the outset of neuropaediatric diseases]. Rev Neurol. 2014 Feb 24;58 Suppl 1:S71-5. Spanish. PMID: 25252671.

[17] Bernaras E, Jaureguizar J, Garaigordobil M. Child and Adolescent Depression: A Review of Theories, Evaluation Instruments, Prevention Programs, and Treatments. Front Psychol. 2019 Mar 20;10:543. doi: 10.3389/fpsyg.2019.00543. PMID: 30949092; PMCID: PMC6435492.

[18] Okereke OI, Singh A. The role of vitamin D in the prevention of late-life depression. J Affect Disord. 2016 Jul 1;198:1-14. doi: 10.1016/j.jad.2016.03.022. Epub 2016 Mar 9. PMID: 26998791; PMCID: PMC4844780.

[19] Williams JA, Romero VC, Clinton CM, Vazquez DM, Marcus SM, Chilimigras JL, Hamilton SE, Allbaugh LJ, Vahratian AM, Schrader RM, Mozurkewich EL. Vitamin D levels and perinatal depressive symptoms in women at risk: a secondary analysis of the mothers, omega-3, and mental health study. BMC Pregnancy Childbirth. 2016 Aug 3;16(1):203. doi: 10.1186/s12884-016-0988-7. PMID: 27485050; PMCID: PMC4971719.

[20] Zhu DM, Zhao W, Zhang B, Zhang Y, Yang Y, Zhang C, Wang Y, Zhu J, Yu Y. The Relationship Between Serum Concentration of Vitamin D, Total Intracranial Volume, and Severity of Depressive Symptoms in Patients With Major Depressive Disorder. Front Psychiatry. 2019 May 9;10:322. doi: 10.3389/fpsyt.2019.00322. PMID: 31143135; PMCID: PMC6520644. 
[21] Shaffer JA, Edmondson D, Wasson LT, Falzon L, Homma K, Ezeokoli N, Li P, Davidson KW. Vitamin D supplementation for depressive symptoms: a systematic review and meta-analysis of randomized controlled trials. Psychosom Med. 2014 Apr;76(3):190-6. doi: 10.1097/PSY.0000000000000044. PMID: 24632894; PMCID: PMC4008710.

[22] Stefanis NC, Hanssen M, Smirnis NK, Avramopoulos DA, Evdokimidis IK, Stefanis CN, Verdoux $\mathrm{H}$, Van Os J. Evidence that three dimensions of psychosis have a distribution in the general population. Psychol Med. 2002 Feb;32(2):347-58. doi: 10.1017/s0033291701005141. PMID: 11866327.

[23] Lichtenstein P, Yip BH, Björk C, Pawitan Y, Cannon TD, Sullivan PF, Hultman CM. Common genetic determinants of schizophrenia and bipolar disorder in Swedish families: a population-based study. Lancet. 2009 Jan 17;373(9659):234-9. doi: 10.1016/S0140-6736(09)60072-6. PMID: 19150704; PMCID: PMC3879718.

[24] Hedelin M, Löf M, Olsson M, Lewander T, Nilsson B, Hultman CM, Weiderpass E. Dietary intake of fish, omega-3, omega-6 polyunsaturated fatty acids and vitamin D and the prevalence of psychoticlike symptoms in a cohort of 33,000 women from the general population. BMC Psychiatry. 2010 May 26;10:38. doi: 10.1186/1471-244X-10-38. PMID: 20504323; PMCID: PMC2889879.

[25] Pilecka I, Sandin S, Reichenberg A, Scragg RKR, David A, Weiderpass E. Sun Exposure and Psychotic Experiences. Front Psychiatry. 2017 Jun 19;8:107. doi: 10.3389/fpsyt.2017.00107. PMID: 28674506; PMCID: PMC5474873.

[26] Ji S, Wang L, Li L. Effect of Metformin on Short-Term High-Fat Diet-Induced Weight Gain and Anxiety-Like Behavior and the Gut Microbiota. Front Endocrinol (Lausanne). 2019 Oct 18;10:704. doi: 10.3389

[27] Salehi-Abargouei A, Esmaillzadeh A, Azadbakht L, Keshteli AH, Afshar H, Feizi A, Feinle-Bisset $\mathrm{C}$, Adibi P. Do patterns of nutrient intake predict self-reported anxiety, depression and psychological distress in adults? SEPAHAN study. Clin Nutr. 2019 Apr;38(2):940-947. doi: 10.1016/j.clnu.2018.02.002.

[28] Kelley L, Sanders AF, Beaton EA. Vitamin D deficiency, behavioral atypicality, anxiety and depression in children with chromosome 22q11.2 deletion syndrome. J Dev Orig Health Dis. 2016 Dec;7(6):616-625. doi: 10.1017/S2040174416000428. PMID: 27827293; PMCID: PMC5922262.

[29] Han B, Zhu FX, Yu HF, Liu S, Zhou JL. Low serum levels of vitamin D are associated with anxiety in children and adolescents with dialysis. Sci Rep. 2018 Apr 13;8(1):5956. doi: 10.1038/s41598-018-24451-7. PMID: 29654252; PMCID: PMC5899084.

[30] Huang JY, Arnold D, Qiu CF, Miller RS, Williams MA, Enquobahrie DA. Association of serum vitamin D with symptoms of depression and anxiety in early pregnancy. J Womens Health (Larchmt). 2014 Jul;23(7):588-95. doi: 10.1089/jwh.2013.4598. Epub 2014 Jun 11. PMID: 24918744; PMCID: PMC4089018.

[31] Kim SY, Jeon SW, Lim WJ, Oh KS, Shin DW, Cho SJ, Park JH, Shin YC. The Relationship between Serum Vitamin D Levels, C-Reactive Protein, and Anxiety Symptoms. Psychiatry Investig. 2020 Apr;17(4):312-319. doi: 10.30773/pi.2019.0290. Epub 2020 Mar 27. PMID: 32213801; PMCID: PMC7176560.

[32] Fazelian S, Amani R, Paknahad Z, Kheiri S, Khajehali L. Effect of Vitamin D Supplement on Mood Status and Inflammation in Vitamin D Deficient Type 2 Diabetic Women with Anxiety: A 
Randomized Clinical Trial. Int J Prev Med. 2019 Feb 12;10:17. doi: 10.4103/ijpvm.IJPVM_174_18. PMID: 30820304; PMCID: PMC6390422.

[33] Ramadan R, Vaccarino V, Esteves F, Sheps DS, Bremner JD, Raggi P, Quyyumi AA. Association of vitamin D status with mental stress-induced myocardial ischemia in patients with coronary artery disease. Psychosom Med. 2014 Sep;76(7):569-75. doi: 10.1097/PSY.0000000000000088. PMID: $25222601 ;$ PMCID: PMC4166546.

[34] Lansdowne AT, Provost SC. Vitamin D3 enhances mood in healthy subjects during winter. Psychopharmacology (Berl). 1998 Feb;135(4):319-23. doi: 10.1007/s002130050517. PMID: 9539254. [35] Choukri MA, Conner TS, Haszard JJ, Harper MJ, Houghton LA. Effect of vitamin D supplementation on depressive symptoms and psychological wellbeing in healthy adult women: a double-blind randomised controlled clinical trial. J Nutr Sci. 2018 Aug 23;7:e23. doi: 10.1017/jns.2018.14. PMID: 30197783; PMCID: PMC6123885.

[36] Penckofer S, Byrn M, Adams W, Emanuele MA, Mumby P, Kouba J, Wallis DE. Vitamin D Supplementation Improves Mood in Women with Type 2 Diabetes. J Diabetes Res. 2017;2017:8232863. doi: 10.1155/2017/8232863. Epub 2017 Sep 7. PMID: 29082262; PMCID: PMC5610883.

[37] Husmann C, Frank M, Schmidt B, Jöckel KH, Antel J, Reissner V, Libuda L, Hebebrand J, Föcker M. Low 25(OH)-vitamin D concentrations are associated with emotional and behavioral problems in German children and adolescents. PLoS One. 2017 Aug 23;12(8):e0183091. doi: 10.1371/journal.pone.0183091. PMID: 28832616; PMCID: PMC5568331.

[38] Dean AJ, Bellgrove MA, Hall T, Phan WM, Eyles DW, Kvaskoff D, McGrath JJ. Effects of vitamin $\mathrm{D}$ supplementation on cognitive and emotional functioning in young adults--a randomised controlled trial. PLoS One. 2011;6(11):e25966. doi: 10.1371/journal.pone.0025966. Epub 2011 Nov 4. PMID: 22073146; PMCID: PMC3208539.

[39] Dumville J.C., Miles J.N., Porthouse J. i wsp.: Can vitamin D supplementation prevent wintertime blues? A randomised trial among older women. J. Nutr. Health Aging 2006; 10: 151-153.

[40] Gloth F.M. 3rd, Alam W., Hollis B.: Vitamin D vs broad spectrum phototherapy in the treatment of seasonal affective disorder. J. Nutr. Health Aging 1999; 3: 5-7.

[41] Hathcock JN, Shao A, Vieth R, Heaney R. Risk assessment for vitamin D. Am J Clin Nutr. 2007 Jan;85(1):6-18. doi: 10.1093/ajcn/85.1.6. PMID: 17209171.

[42] Vieth R. Why the optimal requirement for Vitamin D3 is probably much higher than what is officially recommended for adults. J Steroid Biochem Mol Biol. 2004 May;89-90(1-5):575-9. doi: 10.1016/j.jsbmb.2004.03.038. PMID: 15225842.

[43] Galior K, Grebe S, Singh R. Development of Vitamin D Toxicity from Overcorrection of Vitamin D Deficiency: A Review of Case Reports. Nutrients. 2018 Jul 24;10(8):953. doi: 10.3390/nu10080953. PMID: 30042334; PMCID: PMC6115827. 